\title{
ALMOST ISOMETRIC IDEALS IN BANACH SPACES
}

\author{
TROND A. ABRAHAMSEN \\ Department of Mathematics, Agder University, Servicebox 422, 4604 Kristiansand, Norway \\ e-mail: Trond.A.Abrahamsen@uia.no \\ VEGARD LIMA \\ Ålesund University College, Postboks 1517, 6025 Ålesund, Norway \\ e-mail:Vegard.Lima@gmail.com \\ and OLAV NYGAARD \\ Department of Mathematics, Agder University, Servicebox 422, 4604 Kristiansand, Norway. \\ e-mail: Olav.Nygaard@uia.no
}

(Received 19 October 2012; revised 1 February 2013; accepted 20 June 2013; first published online 13 August 2013)

\begin{abstract}
A natural class of ideals, almost isometric ideals, of Banach spaces is defined and studied. The motivation for working with this class of subspaces is our observation that they inherit diameter 2 properties and the Daugavet property. Lindenstrauss spaces are known to be the class of Banach spaces that are ideals in every superspace; we show that being an almost isometric ideal in every superspace characterizes the class of Gurariy spaces.
\end{abstract}

2010 Mathematics Subject Classification. 46B20, 46B04.

1. Introduction. Let $Y$ be a real Banach space and $X$ a subspace. Recall that $X$ is an ideal in $Y$ if $X^{\perp}$, the annihilator of $X$ in $Y^{*}$, is the kernel of a norm one projection on $Y^{*}$. A linear operator $\varphi: X^{*} \rightarrow Y^{*}$ is called a Hahn-Banach extension operator if $\varphi\left(x^{*}\right)(x)=x^{*}(x)$ and $\left\|\varphi\left(x^{*}\right)\right\|=\left\|x^{*}\right\|$ for all $x \in X$ and $x^{*} \in X^{*}$. If for every finitedimensional subspace $E$ of $Y$ and every $\varepsilon>0$ there exists a linear operator $T: E \rightarrow X$ such that $T e=e$ for all $e \in E \cap X$ and $\|T\| \leq 1+\varepsilon$, then $X$ is said to be locally 1 complemented in $Y$. That these three concepts are just the same thing looked at in three different ways dates back to a 1972 paper by Fakhoury [5]. We will mainly use the locally 1-complemented viewpoint, but following the paper [7] we will use the term ideal. The next theorem can be found in [5, Théorème 2.14] (see also [14, Theorem 3.5]).

THEOREM 1.1. Let $X$ be a subspace of a Banach space $Y$. The following statements are equivalent.

(i) $X$ is an ideal in $Y$.

(ii) There exists a Hahn-Banach extension operator $\varphi: X^{*} \rightarrow Y^{*}$.

(iii) $X$ is locally 1-complemented in $Y$.

The connection between the extension operators and the locally complemented subspaces was further explored in [20]. There the following theorem can be found.

THEOREM 1.2. Let $X$ be a subspace of a Banach space $Y . X$ is an ideal in $Y$ if and only if there exists a Hahn-Banach extension operator $\varphi: X^{*} \rightarrow Y^{*}$ such that for every 
$\varepsilon>0$, every finite-dimensional subspace $E \subset Y$ and every finite-dimensional subspace $F \subset X^{*}$ there exists $T: E \rightarrow X$ such that

(i1) $T e=e$ for all $e \in X \cap E$,

(i2) $\|T e\| \leq(1+\varepsilon)\|e\|$ for all $e \in E$ and

(i3) $\varphi f^{*}(e)=f^{*}(T e)$ for all $e \in E, f^{*} \in F$.

Let us now describe the content of our paper. In searching for a natural condition ensuring that $X$ inherits the property from its superspace $Y$ that every non-void relatively weakly open subset of $B_{Y}$ has diameter 2, we observed that if $T$ in Theorem 1.2 can be assumed to be an $\varepsilon$-isometry, then this diameter 2 property passes down to $X$ from $Y$. This observation is presented in Proposition 3.2. Also, we observed that the same condition works for the problem of inheriting the Daugavet property. The presentation of this result could be found in Proposition 3.8. Precise definitions and necessary background on both diameter 2 properties and the Daugavet property are incorporated in the presentation in Section 3.

The above results on inheriting the diameter 2 property and the Daugavet property indicate that subspaces obeying conclusion in Theorem 1.2 with $T$ almost isometric are of some relevance, and we think it is natural to find out what could be said in general about such subspaces.

Definition 1.3. Let $Y$ be a Banach space and $X$ a subspace. $X$ is called an almost isometric ideal (ai-ideal) in $Y$ if for every $\varepsilon>0$ and every finite-dimensional subspace $E \subset Y$ there exists $T: E \rightarrow X$ which satisfies condition (i1) in Theorem 1.2 and also (ai2) $(1+\varepsilon)^{-1}\|e\| \leq\|T e\| \leq(1+\varepsilon)\|e\|$ for $e \in E$.

Note that $X$ need not be closed in the definition of ai-ideals. By a perturbation argument, a non-closed subspace is an ai-ideal if and only if its closure is also an ai-ideal.

REMARK 1.1. A Banach space $Y$ is finitely representable in $X$ if for every finitedimensional subspace $E \subset Y$ there exists a $T: E \rightarrow X$ such that (ai2) holds.

There is a 1-complemented isometric copy of $\ell_{1}$ in $L_{1}[0,1]$ (see e.g. [4, Lemma 5.1.1]) and, in particular, $\ell_{1}$ is an ideal. $L_{1}[0,1]$ is finitely representable in $\ell_{1}$, but $\ell_{1}$ is not an ai-ideal in $L_{1}[0,1]$ because ai-ideals inherit diameter 2 properties (see Proposition 3.2 below). Hence, there is no $T$ that satisfies properties (i1) and (ai2) simultaneously.

A natural question is whether the analogue of Theorem 1.2 holds. Lindenstrauss' compactness argument, of course, produces a Hahn-Banach extension operator, but the problem is that we risk losing the $\varepsilon$-isometry property of $T$. It turns out that the analogue of Theorem 1.2 is true.

TheORem 1.4. Assume that $X$ is an ai-ideal in $Y$. Then there exists a Hahn-Banach operator $\varphi: X^{*} \rightarrow Y^{*}$ such that for every $\varepsilon>0$, every finite-dimensional subspace $E \subset Y$ and every finite-dimensional subspace $F \subset X^{*}$ there exists $T: E \rightarrow X$ which satisfies statements (i1), (ai2) and (i3).

The proof of this structure result will be the starting point of Section 2.

Note that the conclusion of Theorem 1.4 is very similar to the Principle of Local Reflexivity, and a Banach space $X$ is always an ai-ideal in its bi-dual $X^{* *}$. By Goldstine's theorem, in the Principle of Local Reflexivity setting, the range of $\varphi: X^{*} \rightarrow X^{* * *}$ is 1 -norming for $X^{* *}$ (here $\varphi$ is simply a canonical embedding of $X^{*}$ into $X^{* * *}$ ). We will 
see in Proposition 2.1 that it is in general true that when the range of $\varphi$ in Theorem 1.2 is 1-norming for $Y$, then the ideal $X$ is an ai-ideal in $Y$.

Knowing this, our next question is naturally whether for an ai-ideal the associated Hahn-Banach extension operators $\varphi$ from $X^{*}$ into $Y^{*}$ must have range that is 1norming for $Y$. We will see that this is not so in general; in Example 1 we will see that the 1-co-dimensional subspace $X=\left\{\left(a_{n}\right)_{n=1}^{\infty} \in c_{0}: a_{1}=0\right\}$ of $c_{0}$ is a counterexample.

However, in an important case of u-ideals, the $\varepsilon$-isometry condition of $T$ and having 1-norming range are indeed equivalent (Theorem 2.3). U-ideals were introduced and studied in [7]; we also give some necessary background on u-ideals in the introduction to our Theorem 2.3.

To sum up, our motivation is the question of when diameter 2 properties and the Daugavet property pass to subspaces. This leads to the concept of ai-ideals, which are studied in Section 2, and the results on diameter 2 properties and the Daugavet property form Section 3.

In Section 4 we characterize Gurariy spaces in terms of ai-ideals: It is known from [5] that the Banach spaces that form an ideal in every superspace is exactly the class of Lindenstrauss spaces. We observe in Theorem 4.3 that the class of spaces that are ai-ideals in every superspace is the Gurariy spaces. From this it follows that Gurariy spaces have the Daugavet property. We end the paper by proving that Lindenstrauss spaces in general enjoy diameter 2 properties.

We use standard Banach space notation; symbols and terms will, however, be carefully explained throughout the text when we think it is helpful to the reader. The reader only interested in the results on the passage of diameter 2 properties or the Daugavet property to subspaces may go directly to Section 3.

2. ai-ideals, strict ideals and u-ideals. We start by proving our main structure theorem which was stated in the Introduction.

Proof of Theorem 1.4 We first construct $\varphi$ using a Lindenstrauss compactness argument. Order the set $A=\{(E, F, \varepsilon)\}$, where $E \subset Y$ and $F \subset X^{*}$ are finitedimensional and $\varepsilon>0$ by $\left(E_{1}, F_{1}, \varepsilon_{1}\right) \leq\left(E_{2}, F_{2}, \varepsilon_{2}\right)$ if $E_{1} \subset E_{2}, F_{1} \subset F_{2}$ and $\varepsilon_{2} \leq \varepsilon_{1}$.

For $\alpha \in A, \alpha=(E, F, \varepsilon)$, choose $T_{\alpha}: E \rightarrow X$ satisfying (i1) and (ai2). Define $L_{\alpha}: Y \rightarrow X^{* *}$ by $L_{\alpha} y=T_{\alpha} y$ if $y \in E$ and $L_{\alpha} y=0$ if $y \notin E$. We consider $\left(L_{\alpha}\right) \subset$ $\Pi_{y \in Y} B_{X^{* *}}(0,2\|y\|)$, which by Tychonoff's theorem is compact in the product weak* topology. Without loss of generality, we assume $\left(L_{\alpha}\right)$ is convergent to some $S \in$ $\Pi_{y \in Y} B_{X^{* *}}(0,2\|y\|)$. Note that this implies that for every finite number of elements $\left(y_{i}\right)_{i=1}^{n}$ in $Y$ and $\left(x_{j}^{*}\right)_{j=1}^{m}$ in $X^{*}$ we have

$$
x_{j}^{*}\left(L_{\alpha} y_{i}\right) \rightarrow x_{j}^{*}\left(S y_{i}\right)
$$

By construction, $S y=y$ for every $y \in X$. It is also clear that $\|S\|=1$, hence $\varphi=\left.S^{*}\right|_{X^{*}}$ : $X^{*} \rightarrow Y^{*}$ is a Hahn-Banach extension operator.

Next we apply a perturbation argument modelled after [20, Lemma 1.2], which in turn was inspired by [12].

Let $\left(x_{i}^{*}, x_{i}\right)_{i=1}^{n}$ be a complete bi-orthogonal system for $F$. Define

$$
Q=\sum_{i=1}^{n} i_{X} x_{i} \otimes \varphi\left(x_{i}^{*}\right)
$$


Here $i_{X}: X \rightarrow Y$ is the identity embedding. Then $Q \in \mathcal{F}\left(Y^{*}, Y^{*}\right)$ is a projection with $Q\left(Y^{*}\right)=\varphi(F)$, and $Q^{*}\left(Y^{* *}\right) \subset X$. Similarly, let $P \in \mathcal{F}(E, E)$ be a projection with $P(E)=E \cap X$.

For $\alpha \in A, \alpha=(E, F, \varepsilon)$, let $\left(T_{\alpha}\right)$ be the net from the first paragraph. Define $S_{\alpha}: E \rightarrow X$ by

$$
\begin{aligned}
S_{\alpha} & =i_{E} P+T_{\alpha}\left(I_{E}-P\right)-Q^{*}\left(T_{\alpha}-i_{E}\right)\left(I_{E}-P\right) \\
& =i_{E}+\left(I_{Y^{* *}}-Q^{*}\right)\left(T_{\alpha}-i_{E}\right)\left(I_{E}-P\right) .
\end{aligned}
$$

Here $i_{E}: E \rightarrow Y$ denotes the identity embedding. Now $S_{\alpha} \in \mathcal{F}(E, X)$, because $i_{E} P(E)=E \cap X \subset X$ and $Q^{*}\left(Y^{* *}\right) \subset X$ and $P, T_{\alpha}$, and $i_{E}$ are finite-rank operators. We have $S_{\alpha} e=e$ for every $e \in E \cap X$ because $E \cap X=P(E)$ and $P$ is a projection.

Let $f^{*} \in F$ and $e \in E$. Using $S_{\alpha}(E) \subset X$ we have

$$
\begin{aligned}
\left\langle f^{*}, S_{\alpha} e\right\rangle & =\left\langle\varphi f^{*}, S_{\alpha} e\right\rangle \\
& =\left\langle\varphi f^{*}, i_{E} e\right\rangle+\left\langle\varphi f^{*},\left(I_{Y^{* *}}-Q^{*}\right)\left(T_{\alpha}-i_{E}\right)\left(I_{E}-P\right) e\right\rangle \\
& =\left\langle\varphi f^{*}, i_{E} e\right\rangle+\left\langle\left(I_{Y^{*}}-Q\right) \varphi f^{*},\left(T_{\alpha}-i_{E}\right)\left(I_{E}-P\right) e\right\rangle \\
& =\left\langle\varphi f^{*}, i_{E} e\right\rangle
\end{aligned}
$$

since $Q\left(\varphi f^{*}\right)=\varphi f^{*}$.

So far we have shown that $\left(S_{\alpha}\right)$ satisfies (i1) and (i3). Far out in the net, $S_{\alpha}$ 's will inherit (ai2) from $T_{\alpha}$ 's if we can show that $\left\|S_{\alpha}-T_{\alpha}\right\|$ can be made as small as we wish. Note that

$$
S_{\alpha}-T_{\alpha}=\left(i_{E}-T_{\alpha}\right) P-Q^{*}\left(T_{\alpha}-i_{E}\right)\left(I_{E}-P\right)=-Q^{*}\left(T_{\alpha}-i_{E}\right)\left(I_{E}-P\right)
$$

since $T_{\alpha} e=e$ for all $e \in P(E)$. Thus, we have

$$
\left\|S_{\alpha}-T_{\alpha}\right\|=\sup _{\|e\|=1}\left\|Q^{*}\left(T_{\alpha}-i_{E}\right) e\right\| \leq \sup _{\|e\|=1} \sum_{i=1}^{n}\left\|x_{i}\right\|\left|x_{i}^{*}\left(T_{\alpha} e\right)-\varphi x_{i}^{*}(e)\right| .
$$

Let $\alpha=(E, F, \varepsilon)$. Let $\delta>0$ and choose a $\delta$-net $\left(e_{j}\right)_{j=1}^{k}$ for $S_{E}$. We choose $\beta \geq \alpha$ so that $\left|x_{i}^{*}\left(T_{\beta} e_{j}\right)-\varphi x_{i}^{*}\left(e_{j}\right)\right|<\delta$ for every $i=1, \ldots, n$ and $j=1, \ldots, k$ using (2.1).

For $e \in S_{E}$ choose $j$ such that $\left\|e-e_{j}\right\|<\delta$, then

$$
\begin{aligned}
\left|x_{i}^{*}\left(T_{\beta} e\right)-\varphi x_{i}^{*}(e)\right| & \leq\left|x_{i}^{*}\left(T_{\beta} e\right)-x_{i}^{*}\left(T_{\beta} e_{j}\right)\right|+\left|x_{i}^{*}\left(T_{\beta} e_{j}\right)-\varphi x_{i}^{*}\left(e_{j}\right)\right| \\
& +\left|\varphi x_{i}^{*}\left(e_{j}\right)-\varphi x_{i}^{*}(e)\right| \\
& \leq 2\left\|x_{i}^{*}\right\| \delta+\delta+\left\|x_{i}^{*}\right\| \delta \leq \delta\left(1+3 \max _{i}\left\|x_{i}^{*}\right\|\right) .
\end{aligned}
$$

By choosing $\delta$ small enough, we get that $S_{\beta}$ satisfies (i1), (ai2) and (i3) for the given $\alpha=(E, F, \varepsilon)$. The desired $T: E \rightarrow X$ is then $S_{\beta}$.

In the proof above we used the connection $\varphi=\left.S^{*}\right|_{X^{*}}$ between a Hahn-Banach extension operator $\varphi: X^{*} \rightarrow Y^{*}$ and a norm one extension $S: Y \rightarrow X^{* *}$ of canonical embedding $k_{X}: X \rightarrow X^{* *}$. Clearly, from this connection the existence of a HahnBanach extension operator and a norm one extension of $k_{X}$ to $Y$ are equivalent. Moreover, the existence of a Hahn-Banach extension operator $\varphi: X^{*} \rightarrow Y^{*}$ is equivalent to the existence of a norm one projection $P$ on $Y^{*}$ with $\operatorname{ker} P=X^{\perp}$ and range equal to $\varphi\left(X^{*}\right)$. 
From the way $P, \varphi$ and $S$ are connected, one obtains that the range of $\varphi$ (or $P$ ) is 1 -norming if and only if $S$ is an isometry. This is well studied in the recent literature (see e.g. [21] or [16]); these ideals are called strict ideals.

Proposition 2.1. Suppose $X$ is a strict ideal in $Y$. Then $X$ is an ai-ideal in $Y$.

Proof. Let $\varepsilon>0, E \subset Y$ finite-dimensional and $S: Y \rightarrow X^{* *}$ an isometric extension of $k_{X}$. Since $F=S(E) \subset X^{* *}$ is finite-dimensional, there exists by the Principle of Local Reflexivity $T: F \rightarrow X$ satisfying (i1) and (ai2). It is clear that the composition $T \circ S: E \rightarrow X$ satisfies (i1) and (ai2) and so we are done.

We now give an example that shows the converse of Proposition 2.1 is not true. For this example we will just need a little more background on ideals. An ideal $X \subset Y$ is an $M$-ideal in $Y$ if the ideal projection $P: Y^{*} \rightarrow Y^{*}$ is an L-projection, that is,

$$
\left\|y^{*}\right\|=\left\|P y^{*}\right\|+\left\|y^{*}-P y^{*}\right\| \quad \text { for all } y^{*} \in Y^{*} .
$$

A particular case of this is when $X$ is 1-complemented in $Y$ by an M-projection $Q$, that is, $Q Y=X$ and

$$
\|y\|=\max \{\|Q y\|,\|y-Q y\|\} \quad \text { for all } y \in Y,
$$

in which case $X$ is called an $M$-summand in $Y$. The M-ideal projection is unique (see e.g. [9, Proposition 1.2] or [10, p. 2]). Further, if $X$ is also an M-summand, then $Q^{*}\left(Y^{*}\right)$ is weak* closed, hence if $X$ is a proper subspace of $Y$ it can not be a strict ideal in $Y$.

We denote by $e_{n}$ the $n$th standard basis vector in $c_{0}$ and by $e_{n}^{*}$ its corresponding coordinate functional in $\ell_{1}$.

EXAMPLE 1. The subspace $X=\left\{\left(a_{n}\right)_{n=1}^{\infty} \in c_{0}: a_{1}=0\right\}=\operatorname{ker} e_{1}^{*}$ of $c_{0}$ is 1complemented and an ai-ideal in $c_{0}$.

Proof. Clearly, $X$ is a proper M-summand in $c_{0}$ complemented by the projection $Q$ putting 0 on the first coordinate, and by the above remarks we only need to show that $X$ is an ai-ideal.

Let $E$ be a finite-dimensional subspace of $c_{0}$. and let $\left(x_{i}\right)_{i=1}^{m}$ be some $\varepsilon$-net for $S_{E}$. Find $N$ such that $\left|x_{i}(N)\right|<\varepsilon$ for $i=1,2, \ldots, m$. Define $T: E \rightarrow X$ by $T(y)=$ $Q y+e_{1}^{*}(y) e_{N}$. Then $T$ is obviously linear and an $\varepsilon$-isometry on $\left(x_{i}\right)_{i=1}^{m}$. By [4, Lemma 11.1.11] $T$ is an almost isometry on all of $E$.

As we have seen from Example 1 ai-ideals need not be strict. We will now show that if some symmetry condition is imposed, then ai-ideals indeed are strict. A subspace $X$ is said to be a $u$-ideal in $Y$ if there exists an ideal projection $P: Y^{*} \rightarrow Y^{*}$ such that $\|I-2 P\|=1$ ( $P$ is unconditional). If the range of $P$ is 1 -norming for $Y$, then $X$ is called a strict $u$-ideal in $Y$. There can never be more than one unconditional $P$ ([7, Lemma 3.1]). Further, every M-ideal is a u-ideal. It is known from [7, Proposition 3.6] that $X$ is a $\mathrm{u}$-ideal if and only if $X$ is an ideal with the extra condition

(i4) $\|e-2 T(e)\| \leq(1+\varepsilon)\|e\|$ for all $e \in E$.

We will now assume that $X$ is a u-ideal in $Y$ and $T$ s mentioned above can be chosen to be almost isometries.

Definition 2.2. A subspace $X$ is called an almost isometric u-ideal (ai-u-ideal) if for every $\varepsilon>0$ and every finite-dimensional subspace $E \subset Y$ there exists $T: E \rightarrow X$ which satisfies conditions (i1) and (ai2) and also (i4). 

(i4).

REMARK 2.1. Note that (i2) (and the right-hand inequality of (ai2)) follows from

An inspection of the proof of Theorem 1.4 shows that when $X$ is an ai-u-ideal in $Y$, it is possible to obtain a Hahn-Banach extension operator $\varphi: X^{*} \rightarrow Y^{*}$ which satisfies (i3). However, this observation will not be needed in what follows.

THeOrem 2.3. Let $X$ be a subspace of $Y$. Then $X$ is an ai-u-ideal in $Y$ if and only if $X$ is a strict $u$-ideal in $Y$.

Proof. Assume that $X$ is a strict u-ideal in $Y$. Let $E \subset Y$ and $F \subset X^{*}$ be finite-dimensional subspaces. Let $\phi: X^{*} \rightarrow Y^{*}$ be the strict unconditional extension operator. Let $\mathcal{L}(E, X)$ denote the bounded linear operators from $F$ to $X$ and let $i_{E} \in \mathcal{L}(E, Y)$ be the identity embedding.

Define $\Phi: \mathcal{L}(E, X)^{*} \rightarrow \mathcal{L}(E, Y)^{*}$ by $\Phi\left(e \otimes x^{*}\right)=e \otimes \phi x^{*}$ as in [7, Proposition 3.6]. Using [7, Lemma 2.2] we find a net $\left(T_{\alpha}\right)$ in $\mathcal{L}(F, X)$ converging weak* to $\Phi^{*}\left(i_{E}\right) \in$ $\mathcal{L}(E, X)^{* *}$ such that $\limsup _{\alpha}\left\|i_{E}-2 T_{\alpha}\right\| \leq\left\|i_{E}\right\|=1$. Applying the perturbation argument from [20, Lemma 1.2] (as in Theorem 1.4) we get at linear operator $T: E \rightarrow X$ satisfying (i1), (ai2) and (i4). (For $T$ to become an almost isometry we may have to enlarge $F$.)

Assume that $X$ is an ai-u-ideal in $Y$. Choose $y \in Y \backslash X$. Then $X$ is an ai-uideal in $Z=\operatorname{span}(X,\{y\})$. Let $z \in S_{Z}$ and let $E$ be a finite-dimensional subspace of $Z$ containing $z$. Choose $T: E \rightarrow X^{* *}$ satisfying (i1), (ai2) and (i4). We have $(1-\varepsilon)<$ $(1+\varepsilon)^{-1}$, so by (ai2)

$$
(1-\varepsilon) \leq\|T z\| \leq(1+\varepsilon)
$$

hence

$$
|\|T z\|-1| \leq \varepsilon
$$

Using (i4) we get

$$
\left\|z-2 \frac{T z}{\|T z\|}\right\| \leq\|z-2 T z\|+2\left\|T z-\frac{T z}{\|T z\|}\right\| \leq(1+\varepsilon)+2|1-\|T z\|| \leq 1+3 \varepsilon,
$$

which shows that

$$
\inf _{x \in S_{X}}\|z-2 x\|=1 .
$$

By Theorem 2.4 in [16] $X$ is a strict u-ideal in $Z$. This is true for any $y \in Y$ and so, by Proposition 2.1 in [16], $X$ is a strict u-ideal in $Y$.

3. ai-ideals inherit diameter 2 properties and the Daugavet property. Let $X$ be a nontrivial (real) Banach space with unit ball $B_{X}$. By a slice of $B_{X}$ we mean a set $S\left(x^{*}, \varepsilon\right)=\left\{x \in B_{X}: x^{*}(x)>1-\varepsilon\right\}$, where $x^{*}$ is in the unit sphere $S_{X^{*}}$ of $X^{*}$ and $\varepsilon>0$. A finite convex combination of slices of $B_{X}$ is then a set of the form

$$
S=\sum_{i=1}^{n} \lambda_{i} S\left(x_{i}^{*}, \varepsilon_{i}\right), \quad \lambda_{i} \geq 0, \quad \sum_{i=1}^{n} \lambda_{i}=1,
$$

where $x_{i}^{*} \in S_{X^{*}}$ and $\varepsilon_{i}>0$ for $i=1,2, \ldots, n$. 
The relations between the following three successively stronger properties were investigated in [1].

Definition 3.1. A Banach space $X$ has the

(i) local diameter 2 property if every slice of $B_{X}$ has diameter 2.

(ii) diameter 2 property if every non-empty relatively weakly open subset of $B_{X}$ has diameter 2.

(iii) strong diameter 2 property if every finite convex combination of slices of $B_{X}$ has diameter 2 .

It is not known to us whether properties (i) and (ii) are really different. However, in a recent paper [3] it was established that property (iii) is strictly stronger than property (ii). (The same result has been also independently discovered by R. Haller, J. Langemets, and M. Põldvere, private communication, April 25th 2012). The study of property (ii) above goes back to Shvidkoy's work [22] on the Daugavet property, where a by-product is that spaces with the Daugavet property enjoy the diameter 2 property, and to Nygaard and Werner's paper [19], where uniform algebras are shown to have the diameter 2 property. We postpone the definition of the Daugavet property until needed; it can be found in the introduction to Proposition 3.8 below. A uniform algebra is a separating closed sub-algebra of a $C(K)$-space that contains the constants.

In addition to Daugavet spaces and uniform algebras, spaces with 'big' centralizer are also known to have the diameter 2 property. Precise definition of 'big' centralizer can be found in [1] or [2], we will not give it here as we will not really need it; for our purposes it is enough to know that Daugavet spaces, uniform algebras and spaces with 'big' centralizer form three large classes of spaces with the diameter 2 property.

We believe it is folklore among researchers working on the diameter 2 property that $X$ inherits the diameter 2 property from its bi-dual $X^{* *}$, although we do not know any explicit reference for it. Here we will show the much more general result that all diameter 2 properties are inherited by ai-ideals.

Proposition 3.2. Let $X$ be an ai-ideal in a Banach space $Y$. If $Y$ has the diameter 2 property, so does $X$.

Proof. Let $\varphi$ be the associated Hahn-Banach extension operator from Theorem 1.4. Let $U \subset B_{X}$ be relatively weakly open and $\varepsilon>0$. We will show that for every $x_{0} \in U$ any set of the form

$$
U_{\delta}=\left\{x \in B_{X}:\left|x_{i}^{*}\left(x-x_{0}\right)\right|<\delta, i=1,2, \ldots, n\right\}
$$

contains two points with distance greater than $2-\varepsilon$. Let

$$
V_{\delta}=\left\{y \in B_{Y}:\left|\varphi x_{i}^{*}\left(y-x_{0}\right)\right|<\delta, i=1,2, \ldots, n\right\} .
$$

$V_{\delta}$ is relatively weakly open in $B_{Y}$ and hence has diameter 2 . Thus, we can find $z_{1}, z_{2} \in V_{\delta}$ with $\left\|z_{1}\right\| \leq 1,\left\|z_{2}\right\| \leq 1$ and $\left\|z_{1}-z_{2}\right\|>2-\varepsilon / 4$. Let $0<\eta<\varepsilon / 8$ and set $y_{i}=(1+\eta)^{-1} z_{i}$. Then $\left\|y_{1}-y_{2}\right\|>2-\varepsilon / 2$.

Let $E=\operatorname{span}\left\{x_{0}, y_{1}, y_{2}\right\}$ and $F=\operatorname{span}\left\{x_{i}^{*}\right\}_{i=1}^{n}$. Use Theorem 1.4 to find an $\eta$ isometry $T: E \rightarrow X$. Then $\left\|T y_{i}\right\| \leq(1+\eta)\left\|y_{i}\right\| \leq 1$,

$$
\left\|T y_{1}-T y_{2}\right\| \geq(1+\eta)^{-1}\left\|y_{1}-y_{2}\right\|>2-\varepsilon,
$$


and for $i=1,2, \ldots, n$ and $j=1,2$ we have

$$
\left|x_{i}^{*}\left(T y_{j}-x_{0}\right)\right|=\left|x_{i}^{*}\left(T y_{j}-T x_{0}\right)\right|=\left|x_{i}^{*}\left(T\left(y_{j}-x_{0}\right)\right)\right|=\left|\varphi x_{i}^{*}\left(y_{j}-x_{0}\right)\right|<\delta,
$$

hence $T y_{1}, T y_{2} \in U_{\delta}$.

REMARK 3.1. Note that in the proof above we only needed to push every threedimensional $E \subset Y$ into $X$ almost isometrically.

Now we prove that the strong diameter 2 property is also inherited by ai-ideals.

Proposition 3.3. Let $X$ be an ai-ideal in Banach space $Y$. If $Y$ has the strong diameter 2 property, so does $X$.

Proof. Let $S \subset B_{X}$ be a finite convex combination of slices. $S$ is then of the form

$$
S=\sum_{i=1}^{n} \lambda_{i} S_{i}\left(x_{i}^{*}, \varepsilon_{i}\right),
$$

where $x_{i}^{*} \in B_{X}^{*}, \varepsilon_{i}>0, \lambda_{i}>0$ and $\sum_{i=1}^{n} \lambda_{i}=1$. Now put

$$
S_{\varphi}=\sum_{i=1}^{n} \lambda_{i} S_{\varphi, i}\left(\varphi x_{i}^{*}, \varepsilon_{i}\right),
$$

where $\varphi$ is the Hahn-Banach extension operator associated with an ai-ideal. Note that each $S_{\varphi, i}\left(\varphi x_{i}^{*}, \varepsilon_{i}\right)=\left\{y \in B_{Y}: \varphi x_{i}^{*}(y)>1-\varepsilon_{i}\right\}$ is a slice of $B_{Y}$. Since $S_{\varphi}$ has diameter 2 , there are for every $\eta>0, y_{k} \in S_{\varphi}, k=1,2$, such that $\left\|y_{1}-y_{2}\right\|>2-\eta$. Now $y_{k} \in S_{\varphi}$ is of the form $y_{k}=\sum_{i=1}^{n_{k}} \lambda_{i} y_{\varphi, k}^{i}$, where $y_{\varphi, k}^{i} \in S_{\varphi, i}\left(\varphi x_{i}^{*}, \varepsilon_{i}\right)$. Let $E=$ $\operatorname{span}\left(y_{k}, y_{\varphi, k}^{n_{k}}\right)_{k, i} \subset Y$ and $F=\operatorname{span}\left(x_{i}^{*}\right)_{i} \subset X^{*}$. By a perturbation argument, we may assume that $\max _{k}\left\|y_{k}\right\|=r<1$.

For $\delta>0$ such that $(1+\delta) \cdot r \leq 1$, choose $T: E \rightarrow X$ which fulfills (i)-(iii) in the conclusion of Theorem 1.4 with this $\delta$, and observe that $T y_{k}=\sum_{i=1}^{n_{k}} \lambda_{i} T y_{\varphi, k}^{i}$. Then $T y_{k} \in S$ since $\left\|T y_{k}\right\| \leq(1+\delta)\left\|y_{k}\right\| \leq(1+\delta) \cdot r \leq 1$, and $T y_{\varphi, k}^{i}\left(x_{i}^{*}\right)=\varphi x_{i}^{*}\left(y_{\varphi, k}^{i}\right)>1-$ $\varepsilon_{i}$, so $T y_{\varphi, k}^{i} \in S_{i}$.

Finally, observe that $\left\|T y_{1}-T y_{2}\right\|>(1+\delta)^{-1}(2-\eta)$ and $\delta$ and $\eta$ may be chosen arbitrarily small, so the diameter of $S$ must be 2 .

REMARK 3.2. Note that in the proof above we cannot take $E$ with just three dimensions as we could in the proof of the similar result for the diameter 2 property.

COROLlary 3.4. Almost isometric ideals inherit the local diameter 2 property.

Proof. Take $n=1$ in the proof of Proposition 3.3.

Proposition 3.5. Let $Y$ be a Banach space. If every infinite-dimensional separable ideal in $Y$ has the (local, strong) diameter 2 property, so does $Y$.

Proof. Firstly, let us prove the result for the strong diameter 2 property. To this end let $\varepsilon_{i}>0$ for $i=1, \ldots, n$ and $S=\sum_{i=1}^{k} \lambda_{i} S_{i}$, a finite convex combination of slices $S_{i}=\left\{y \in B_{Y}: y_{i}^{*}(y)>1-\varepsilon_{i}\right\}$ of the unit ball of $Y$. Each slice is relatively weakly open and therefore contains a ball of small radius about a point in the slice. Thus, it is possible to find a sequence of infinitely many linearly independent points in each slice. It is clearly also possible to find a linearly independent sequence $\left(y_{n}\right) \subset S$. Let $Z$ be the 
norm closure of $\operatorname{span}\left(y_{n}\right)$. By [11] (cf. [10, Lemma III.4.3]) there is a separable ideal $X$ in $Y$ containing $Z$ such that $\operatorname{span}\left(y_{i}^{*}\right)_{i=1}^{k} \subset \varphi\left(X^{*}\right)$, where $\varphi: X^{*} \rightarrow Y^{*}$ is the HahnBanach extension operator. Now, for $i=1, \ldots, k$, find $x_{i}^{*} \in X^{*}$ such that $y_{i}^{*}=\varphi\left(x_{i}^{*}\right)$. Let $S_{i}^{\prime}=\left\{x \in B_{X}: x_{i}^{*}(x)>1-\varepsilon_{i}\right\}=\left\{x \in B_{X}: \varphi x_{i}^{*}(x)>1-\varepsilon_{i}\right\}$ be slices of the unit ball of $X$. Denote by $S^{\prime}=\sum_{i=1}^{k} \lambda_{i} S_{i}^{\prime}$ the corresponding convex combination of slices. Since $S^{\prime}$ has diameter 2 and $S^{\prime} \subset S, S$ has diameter 2 .

For the local diameter 2 property the result follows by taking $k=1$ in the argument above.

For the diameter 2 property let $V$ be a relatively weakly open subset in $B_{Y}$. Find $y_{0} \in V$ and $y_{i}^{*} \in Y^{*}$ such that $V_{\varepsilon}=\left\{y \in B_{Y}:\left|y_{i}^{*}\left(y-y_{0}\right)\right|<\varepsilon, i=1, \cdots, n\right\} \subset V$. It is possible to choose a sequence $\left(y_{n}\right)$ of infinitely many linearly independent points in $V_{\varepsilon}$ and a similar argument as above will now finish the proof.

Our next goal is to show that ai-ideals inherit the Daugavet property. Let us first recall the definition of this property.

DEFINITION 3.6. A Banach space $X$ has the Daugavet property if for every rank 1 operator $T: X \rightarrow X$,

$$
\|T+I\|=1+\|T\| .
$$

In Definition 3.6, $I$ denotes the identity operator on $X$. We will need a fundamental observation from [13, Lemma 2.2].

LEMMA 3.7. The following are equivalent.

(i) $X$ has the Daugavet property.

(ii) For all $y \in S_{X}, x^{*} \in S_{X^{*}}$ and $\varepsilon>0$, there exists $x \in S_{X}$ such that $x^{*}(x) \geq 1-\varepsilon$ and $\|x+y\| \geq 2-\varepsilon$.

The next result is proved for M-ideals in [13, Proposition 2.10].

Proposition 3.8. If $X$ is an ai-ideal in $Y$ and $Y$ has the Daugavet property, then $X$ has the Daugavet property.

Proof. Let $\varphi: X^{*} \rightarrow Y^{*}$ be the (ai-) Hahn-Banach extension operator.

We will show that (ii) in Lemma 3.7 is fulfilled. For this, let $y \in S_{X}, x^{*} \in S_{X^{*}}$ and $\varepsilon>0$. Consider the slice

$$
S_{1}=\left\{x \in B_{X}: x^{*}(x) \geq 1-\varepsilon\right\} .
$$

We will need to produce some $x \in S_{1}$ with $\|x\|=1$ and $\|y+x\| \geq 2-\varepsilon$. Look at

$$
S=\left\{z \in B_{Y}: \varphi\left(x^{*}\right)(z) \geq 1-\eta\right\}
$$

Since $Y$ has the Daugavet property, for all $\eta>0$, there is some $z \in S$ with $\|z\|=1$ such that $\|z+y\| \geq 2-\eta$. Let $\frac{\varepsilon}{2}>\eta>0$ and choose $\frac{\varepsilon}{2}>\delta>0$ such that $\delta \leq \frac{\frac{\varepsilon}{2}-\eta}{2-\frac{\varepsilon}{2}}$. Note that this choice gives $(1+\delta)^{-1}(2-\eta) \geq 2-\frac{\varepsilon}{2}$.

Let $E=\operatorname{span}\{z, y\} \subseteq Y, F=\operatorname{span}\left\{x^{*}\right\} \subseteq X^{*}$ and find a corresponding $\delta$-isometry $T: E \rightarrow X$. Let $x=\frac{T(\bar{z})}{\|T(z)\|}$. Clearly, $\|x\|=1$. We get

$$
\|x-T(z)\|=|\|T(z)\|-1| \leq \delta \leq \frac{\varepsilon}{2},
$$


hence

$$
\|x+y\| \geq\|T(z)+y\|-\|x-T(z)\| \geq(1+\delta)^{-1}(2-\eta)-\delta \geq 2-\varepsilon .
$$

Finally,

$$
x^{*}(x)=x^{*}(T(z))+x^{*}(x-T(z)) \geq \varphi\left(x^{*}\right)(z)-\delta \geq 1-\eta-\delta \geq 1-\varepsilon,
$$

and we conclude that $X$ has the Daugavet property.

REMARK 3.3. As in the proof of Proposition 3.2, the full strength of an ai-ideal was not needed in the above proof. We only needed $E$ 2-dimensional and $F$ 1-dimensional. Of course, as for diameter 2 properties, we also get from Proposition 3.8 that $X$ inherits the Daugavet property from $X^{* *}$, but this is trivial since, from the definition of the Daugavet property, $X$ always has the Daugavet property if $X^{*}$ does.

Recall Milne's theorem that every Banach space is a 1-complemented subspace of a uniform algebra. Wojtasczyk observed in [25, Corollary 4] that the standard proof of this theorem yields a uniform algebra with the Daugavet property, hence also the strong diameter 2 property. In particular, diameter 2 properties do not automatically pass to 1-complemented subspaces and hence not to ideals.

4. Gurariy-spaces in terms of ai-ideals. Recall that a Lindenstrauss space is a Banach space such that the dual is an $L_{1}(\mu)$-space for some (positive) measure $\mu$. Fakhoury [5, Proposition 3.4] has proved the following result.

THEOREM 4.1. For a Banach space $X$ the following statements are equivalent:

(i) $X$ is a Lindenstrauss space.

(ii) $X$ is an ideal in every superspace.

Now we will prove an analogous result for ai-ideals. For this we will need the definition of Gurariy space.

Definition 4.2. A Banach space $X$ is called a Gurariy space if it has the property that whenever $\varepsilon>0, E$ is a finite-dimensional Banach space, $T_{E}: E \rightarrow X$ is isometric and $F$ is a finite-dimensional Banach space with $E \subset F$, then there exists a linear operator $T_{F}: F \rightarrow X$ such that

(i) $T_{F}(f)=T_{E}(f)$ for all $f \in E$, and

(ii) $(1+\varepsilon)^{-1}\|f\| \leq\left\|T_{F} f\right\| \leq(1+\varepsilon)\|f\|$ for all $f \in F$.

If $T_{F}: F \rightarrow X$ is taken to be isometric, then $X$ is called a strong Gurariy space.

Gurariy proved in [8] that Gurariy spaces exist. Indeed, he constructed a separable such Banach space and showed that all separable Gurariy spaces are linearly almost isometric. Later Lusky [18] proved that all separable Gurariy spaces are in fact linearly isometric. The fact that strong Gurariy spaces exist can be found in [6].

Let us now state and prove a result similar to Theorem 4.1 for ai-ideals.

THEOREM 4.3. For a Banach space $X$, the following statements are equivalent:

(i) $X$ is a Gurariy space.

(ii) $X$ is an ai-ideal in every superspace.

Proof. (i) $\Rightarrow$ (ii). Let $X$ be a subspace of $Y, E$ a finite-dimensional subspace of $Y$ and $\varepsilon>0$. If $E \cap X$ is of dimension $\geq 1$, then $T: E \cap X \rightarrow X$ is the identity operator. 
By assumption, there is a linear extension $\hat{T}$ of $T$ satisfying $(1+\varepsilon)^{-1}\|e\| \leq\|\hat{T} e\| \leq$ $(1+\varepsilon)\|e\|$ for every $e \in E$, just as needed. Now, if $E \cap X=\{0\}$, then choose some non-zero $x \in X$, put $E^{\prime}=\operatorname{span}(E,\{x\})$ and argue as above.

(ii) $\Rightarrow$ (i). Let $\varepsilon>0$ and choose $\delta>0$ such that $(1+\delta)^{2} \leq 1+\varepsilon$. By [6, Theorem 3.6] we can assume that $X$ is a subspace of a Gurariy space $X_{G}$. Now, let $E \subset F$ be finite-dimensional subspaces, and $T: E \rightarrow X$ be linear and isometric. Since $X_{G}$ is a Gurariy space, there exists a linear extension $\hat{T}: F \rightarrow X_{G}$ of $T$ with $(1+\delta)^{-1}\|f\| \leq$ $\|T(f)\| \leq(1+\delta)\|f\|$ for every $f \in F$. Put $H=\hat{T}(F)$. Since $X$ is an ai-ideal in $X_{G}$, there exists an operator $S: H \rightarrow X$ satisfying $(1+\delta)^{-1}\|h\| \leq\|S h\| \leq(1+\delta)\|h\|$ for every $h \in H$ such that $S h=h$ for every $h \in H \cap X$. It follows that $S \circ \hat{T}: F \rightarrow X$ is a linear extension of $T$ satisfying $(1+\varepsilon)^{-1}\|f\| \leq\|S \circ \hat{T}(f)\| \leq(1+\varepsilon)\|f\|$ for every $f \in F$, which is exactly as desired.

REMARK 4.1. It follows from the techniques used in [6] that every non-separable Banach space can be isometrically embedded in a strong Gurariy space. Thus, by arguing as in Theorem 4.3, it is easily seen that strong Gurariy spaces are exactly the spaces that are ai-ideals with $\varepsilon=0$ in every superspace.

COROLlary 4.4. The separable Gurariy space is the only separable Banach space that is an ai-ideal in every superspace.

The Daugavet property for Lindenstrauss spaces was studied by Werner (see [23, Theorem 3.5]). We note the following.

COROLlary 4.5. Gurariy spaces enjoy the Daugavet property and hence the strong diameter 2 property.

Proof. Let $X$ be a Gurariy space. $C\left(B_{X^{*}}\right.$, weak $\left.{ }^{*}\right)$ has the Daugavet property (cf. e.g. [24]), and since $X$ embeds isometrically into $C\left(B_{X^{*}}\right.$, weak $\left.{ }^{*}\right)$, the result follows Theorem 4.3 and Proposition 3.8.

It is clear from Theorems 4.1 and 4.3 that a Gurariy space is a Lindenstrauss space. Thus, the last part of Corollary 4.5 is also a particular case of the following result (see also [3, Corollary 3.6]).

Proposition 4.6. The bi-dual of every infinite-dimensional Lindenstrauss space has the strong diameter 2 property. In particular, every Lindenstrauss space has the strong diameter 2 property.

Proof. Let $X$ be a Lindenstrauss space. It is classical that $X^{*}$ is order isometric to an $\ell_{1}$-sum of $L_{1}\left(\mu_{a}\right)$-spaces, where $\mu_{a}$ is a probability measure (see e.g. [17, Theorem 1.b.2]).

Now there are two possibilities. Either every $\mu_{a}$ is purely atomic, and then $X^{*}$ is isometric to $\ell_{1}(\Gamma)$ for some set $\Gamma$, or one $\mu_{a}$ is not purely atomic (see [15, Theorem 5.14.9] for a concrete representation). In the first case $X^{* *}=\ell_{\infty}(\Gamma)$, which has the strong diameter 2 property. In the latter case we may write $X^{* *}=Z \oplus_{\infty} L_{\infty}\left(\mu_{a}\right)$ and thus $X^{* *}$ has the strong diameter 2 property by Proposition 4.6 in [1]. In either case $X^{* *}$ has the strong diameter 2 property. 
Note that not all Lindenstrauss spaces, e.g. $c_{0}$, have the Daugavet property, see also [24, p. 79].

ACKNOWLEDGEMENTS. We would like to thank Wiesław Kubiś for fruitful discussions on the topic of Section 4.

\section{REFERENCES}

1. T. A. Abrahamsen, V. Lima and O. Nygaard, Remarks on diameter 2 properties, $J$. Conv. Anal. 20 (2) (2013), 439-452.

2. M. D. Acosta and J. Becerra Guerrero, Weakly open sets in the unit ball of some Banach spaces and the centralizer, J. Funct. Anal. 259 (4) (2010), 842-856. MR 2652174 (2011e:46078).

3. M. D. Acosta, J. Becerra Guerrero and G. López-Pérez, Stability results of diameter two properties, J. Convex Anal. (to appear).

4. F. Albiac and N. J. Kalton, Topics in Banach space theory, Graduate Texts in Mathematics, vol. 233 (Springer, New York, 2006). MR 2192298 (2006h:46005).

5. H. Fakhoury, Sélections linéaires associées au théorème de Hahn-Banach, J. Funct. Anal. 11 (1972), 436-452. MR 0348457 (50 \#955). $235-269$.

6. J. Garbulinśka and W. Kubiś, Remarks on Gurariǐ spaces, Extracta Math. 26 (2) (2011),

7. G. Godefroy, N. J. Kalton and P. D. Saphar, Unconditional ideals in Banach spaces, Studia Math. 104 (1) (1993), 13-59. MR 1208038 (94k:46024).

8. V. I. Gurariî, Spaces of universal placement, isotropic spaces and a problem of Mazur on rotations of Banach spaces, Sibirsk. Mat. Ž. 7 (1966), 1002-1013. MR 0200697 (34 \#585).

9. P. Harmand and $\AA$. Lima, Banach spaces which are $M$-ideals in their biduals, Trans. Amer. Math. Soc. 283 (1) (1984), 253-264. MR 735420 (86b:46016).

10. P. Harmand, D. Werner and W. Werner, $M$-ideals in Banach spaces and Banach algebras, Lecture Notes in Mathematics, vol. 1547 (Springer-Verlag, Berlin, Germany, 1993). MR 1238713 (94k:46022).

11. S. Heinrich and P. Mankiewicz, Applications of ultrapowers to the uniform and Lipschitz classification of Banach spaces, Studia Math. 73 (3) (1982), 225-251. MR 675426 (84h:46026).

12. W. B. Johnson, H. P. Rosenthal and M. Zippin, On bases, finite dimensional decompositions and weaker structures in Banach spaces, Israel J. Math. 9 (1971), 488-506. MR 0280983 (43 \#6702).

13. V. M. Kadets, R. V. Shvidkoy, G. G. Sirotkin and D. Werner, Banach spaces with the Daugavet property, Trans. Amer. Math. Soc. 352 (2) (2000), 855-873. MR 1621757 (2000c:46023).

14. N. J. Kalton, Locally complemented subspaces and $\mathcal{L}_{p}$-spaces for $0<p<1$, Math. Nachr. 115 (1984), 71-97. MR 755269 (86h:46006).

15. H. E. Lacey, The isometric theory of classical Banach spaces, Die Grundlehren der mathematischen Wissenschaften, Band 208 (Springer-Verlag, New York, 1974). MR 0493279 (58 \#12308).

16. V. Lima and Å. Lima, Strict u-ideals in Banach spaces, Studia Math. 195 (3) (2009), 275-285. MR 2559177 (2010g:46014).

17. J. Lindenstrauss and L. Tzafriri, Classical Banach spaces. II, Ergebnisse der Mathematik und ihrer Grenzgebiete (Results in Mathematics and Related Areas), vol. 97 (Springer-Verlag, Berlin, Germany, 1979), Function spaces MR 540367 (81c:46001).

18. W. Lusky, The Gurarij spaces are unique, Arch. Math. (Basel) 27 (6) (1976), 627-635. MR 0433198 ( 55 \#6177).

19. O. Nygaard and D. Werner, Slices in the unit ball of a uniform algebra, Arch. Math. (Basel) 76 (6) (2001), 441-444. MR 1831500 (2002e:46057).

20. E. Oja and M. Põldvere, Principle of local reflexivity revisited, Proc. Amer. Math. Soc. 135 (4) (2007), 1081-1088 (electronics). MR 2262909.

21. T. S. S. R. K. Rao, On ideals in Banach spaces, Rocky Mountain J. Math. 31 (2) (2001), 595-609. MR 1840956 (2002d:46018). 
22. R. V. Shvydkoy, Geometric aspects of the Daugavet property, J. Funct. Anal. 176 (2) (2000), 198-212. MR 1784413 (2001h:46019).

23. D. Werner, The Daugavet equation for operators on function spaces, J. Funct. Anal. 143 (1) (1997), 117-128. MR 1428119 (98c:47025).

24. D. Werner, Recent progress on the Daugavet property, Irish Math. Soc. Bull. (46) (2001), 77-97. MR 1856978 (2002i:46014).

25. P. Wojtaszczyk, Some remarks on the Daugavet equation, Proc. Amer. Math. Soc. 115 (4) (1992), 1047-1052. MR 1126202 (92k:47041). 